\title{
Tongue reshaping: a new surgical method to prevent self-sucking in dairy cows
}

\author{
Mohamed Wefky El-Sherif* \\ Department of surgery, Faculty of Veterinary Medicine, Assiut University, New Valley, El-Kharga, Egypt
}

\begin{abstract}
Self-sucking behaviors lead to substantial milk loss and inflammation of the udder and teats among dairy cows. Both conservative and surgical approaches are available to prevent such behavior; however, the current methods are limited by variable efficacy and risk of complications. The objective of the present study was to evaluate a new minimally invasive technique for reshaping of the tongue to prevent self-sucking. The current technique was performed on five mixed breed dairy cows aged 4-6 years and with a mean weight $320 \mathrm{kgs}$. Reshaping of the normal contour of the tongue was achieved by the application of multiple interrupted inverting sutures to the ventral surface to prevent the tongue from forming "U" shape. Post-operative approach included antiseptic mouth wash with povidone iodine solution twice daily and recording the healing process and complications. The mean operative time was $12 \pm 1$ minutes. No complications except superficial pressure wounds from $3^{\text {rd }}$ up to $10^{\text {th }}$ day post-operative were observed. At three months follow up all treated cows had stopped self-sucking. One-shot tongue reshaping is an easy, minimally invasive, effective, and time-saving procedure for the treatment of self-sucking among dairy cows, with the advantage of causing little tissue damage.
\end{abstract}

Keywords: Cattle, Glossectomy, Self-sucking.

\section{Introduction}

Self-sucking of the teats is a behavioral problem among dairy cows that results in severe economic losses to the dairy industry owing to reduced milk production and increased need for veterinary care (Boe, 1990; Debreceni and Juhas, 1999). Regardless of the causes of self-sucking behavior, the affected animals shape their tongue like a cup and suck their own teats (Boe, 1990). This abnormal behavior results in several serious complications in affected animal such as teat injuries, mastitis and loss of milk (Bademkiran et al., 2006). Several approaches are available to treat this condition. Where the self-sucking behavior is extensively found in an area; searching for definitive, reliable treatment is advisable.

Conservative treatments include application of nose rings with spikes (Allmacher, 1998; Abou-El-Ella, 1999; Bademkiran et al., 2006), rings fixed to the frenulum linguae (Allmacher, 1998) and application of neck cradles (Bademkiran et al., 2006).

Conservative treatments do not resolve the condition (Abou-El-Ella, 1999; Bademkiran et al., 2006). Some injuries can result from devices applied to control selfsucking. A nose ring with spikes can cause serious injuries to both the affected animal and to other animals. Neck cradles also cause injuries to the neck of the affected animal (Bademkiran et al., 2007). Animal with self-sucking preventing device is easily recognized and became market inferior.
In terms of outcome, surgical intervention offers a more effective approach for prevention of self-sucking behavior than does the use of conservative treatments (Abou-El-Ella, 1999). Surgical techniques include partial glossectomy (Lyle, 1971; Ducharme, 2004), transection of the ventral lingual mucosa (also known as ventral glossectomy) (McCormack, 1976; Pamer, 1981; Abou-El-Ella, 1999; Ducharme, 2004; Yong et al., 2008) and full-thickness glossectomy (Abou-ElElla, 1999). Partial glossectomy involves transection of approximately 2 inches of a full-thickness portion of the tongue, from either the tip or the lateral aspect, followed by suturing incisions to preserve the tongue shape (Ducharme, 2004). Ventral glossectomy involves an elliptical incision that is approximately $5 \mathrm{~cm}$ at its widest part, starts rostral to the frenulum attachment on the tongue and extends rostrally $2.5 \mathrm{~cm}$ caudal to the tip of the tongue. By suturing the ellipse, the dorsal surface of the tongue becomes convex and is unable to form the "U" shape required for self-sucking of the teat (Ducharme, 2004). Full-thickness glossectomy involves the removal of an elliptical full-thickness area from the ventral aspect of the tongue. This method provided similar results to ventral glossectomy (AbouEl-Ella, 1999).

The previously mentioned glossectomy techniques require surgical excision of variable thickness of the tongue in order to disrupt its contour and prevent the tongue from curling so that suction is not possible. Such 
techniques may require general anesthesia (Lyle, 1971), time consuming and involves tissue damage with at most subsequent bleeding and sepsis.

Surgical techniques score high when compared with the conservative techniques, but, presence of a new approach implements disruption of the contour of the tongue with minimal tissue invasion and post-operative complications will be an addition. Consequently, the aim of present study was to evaluate a new minimally invasive surgical technique (one-shot tongue reshaping) for the treatment of self-sucking behavior among dairy cows.

\section{Materials and Methods \\ Patient animal Presentation}

Five mixed-breed dairy cows aged 4-6 years and with a mean weight of $320 \mathrm{~kg}$ were referred by local veterinarians from multiple farms with a vice of selfsucking of milk. Two procedures were performed within one week during the act of the annual mobile clinic held by the faculty of veterinary medicine, New Valley branch, the other three cases were performed sporadically and within wide range of time. Animal's histories were thoroughly collected, farmers were asked for; how they discover the problem, did they bear witness cows with the action, did they use conservative measure, and if yes, what are these methods and for what period did they use it, and if it was effective or not? All farmers referred their cows for surgery for the good results obtained in similar problem by other farmers. The new experimental technique was explained to the owners and declaration to perform was obtained before surgery.

\section{Pre-operative measures}

Physiological parameters, including rectal temperature, heart rate and respiratory rate, were evaluated before surgery to decide whether an individual cow was sufficiently healthy to undergo surgery.

Cows were casted with ropes and placed in lateral recumbency at any side on a soft padded area. In order to avoid excessive salivation and tympany, sedatives were not used. An assistant was asked to secure the head by pressing the basal septum with a hand and manually grasp and pull the tongue out of the mouth. A strip of gauze was placed to the most caudal part of the tongue and tied well as tourniquet, the ends of the gauze were tied over the head to keep the tongue in place out of the tongue.

\section{Procedure}

The ventral aspect of the tongue is exposed to face the operator and the tip of the tongue is grasped with atraumatic forceps. Five $\mathrm{ml}$ of $2 \%$ lignocaine hydrochloride is infiltrated $1 \mathrm{~cm}$ lateral on either side of median raphae of tongue.

Instruments required were as follows: half circle reverse cutting size (5-6) needle, non-absorbable suture material of size (3-4) (Braided Silk, Pearsalls ltd, England), needle holder and a scissor.

The ventral aspect of the tongue is cleaned with sterile isotonic saline solution and dried with sterile towel before suturing.

Each single interrupted stitch is made as follow: A bite $(0.5 \mathrm{~cm})$ including the mucosa and deep into the muscle is taken on one side of the tongue $0.5 \mathrm{~cm}$ lateral to its midline with the direction of needle perpendicular to midline of the tongue, crossing over to the other side of the tongue and another bite is taken in the same direction, a square knot is tied twice, edges are inverted immediately. Each stitch is placed $0.5 \mathrm{~cm}$ apart. Then, going onto the same side where you started and do the same. Through the tissue the suture runs perpendicular to the tongue midline while the exposed portion of the suture runs diagonal to its midline (Figure 1-A, B, C). The first stitch is placed at the most caudal portion of ventral aspect of the tongue nearby the frenulum linguae, then, stitching is advanced cranially. The last stitch is placed about $3 \mathrm{~cm}$ caudal to the tip of the tongue. The stitches are checked for prober tightness and that no gap left between the edges of the tongue (Figure 1-D). The midline portion of the tongue where the lingual blood vessels lie is not invaded by the needle.

The tongue is irrigated again with sterile saline solution and betadine mouth wash. The tourniquet is removed and the tongue is checked for bleeding or other complications. The contour of the tongue is checked for its new modification and the tongue is not able to form the "U" shape for sucking. Then, the anesthetized tongue is replaced into the mouth cavity and the cow is permitted to stand up.
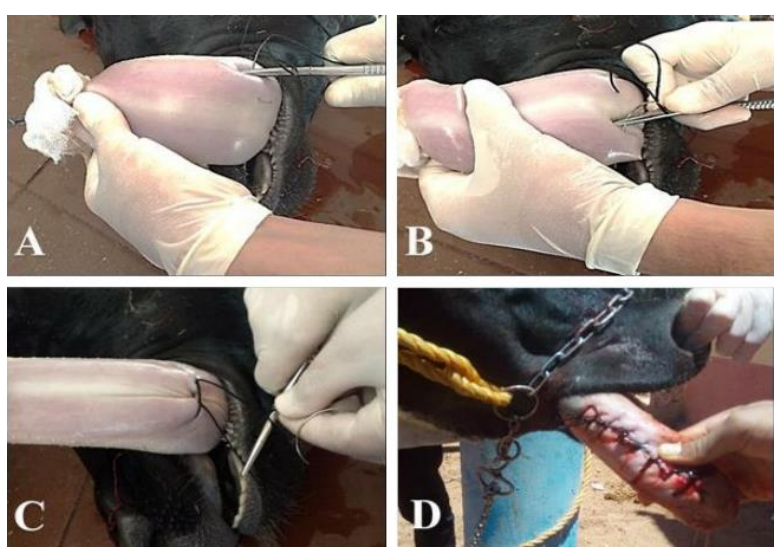

Fig. 1. Steps of the suturing process during one-shot tongue reshaping. (A): Application of the first bite to one side of the midline of the tongue. (B): The needle is crossed over and a second bite is taken at the opposite side of the tongue. (C): The suture is tied in square knot. (D): Multiple interrupted inverting sutures are applied starting at the base and advanced toward the tip of the tongue. 


\section{Post-operative measures}

An adhesive tape is used to close the mouth for three hours after the procedure in order to prevent protrusion of the anesthetized tongue until anesthesia effect is over.

Owners were advised to offers the cows soft palatable food, isolation of the cow, irrigation of the mouth with povidone iodine solution (Betadine -mouth wash, Alkahirapharma, Egypt) twice daily and systemic antibiotic and anti-inflammatory are administered for seven successive days.

All cows were refereed to local veterinarian for daily examination of the tongue and disappearance of selfsucking. The tongue was examined for persistence of the new contour, condition of the sutures, postoperative macro changes and post-operative complications. Sutures were designed to left in place and not removed to keep the new tongue contour.

Outcome measures included the mean operative time; intraoperative complications; degree of tissue destruction; healing time; and postoperative complications.

\section{Results}

\section{Pre-operative}

Physiological parameters in all presented cows were sufficiently normal for performing surgery. All vital parameters remained in normal physiological range after performing the procedure.

\section{Intra-operative}

Slight bleeding occurred at the puncture points of needle insertion; however, no other intraoperative complications were noted. The lingual blood vessels and nerve at the midline portion of the tongue were not invaded and remained intact.

\section{Post-operative}

The mean operative time was $12 \pm 1$ minutes. The immediate result of applying multiple inverting sutures to the ventral surface of the tongue was disruption of the normal contour of the tongue. The dorsal surface of the tongue became more convex and the tongue does not have the ability to form the "U" shape.

No further bleeding was noted after removal of the tourniquet. Swelling of the ventral aspect of the tongue was observed among all five cows on the day after surgery. The stitches had caused pressure wounds by the third postoperative day; these wounds were still apparent at 7-10 days (Fig. 2-A).

Stitches were buried and complete healing was recorded at 14-16 days after the procedure (Fig. 2-B). Application of an adhesive elastic bandage to the tongue was required for one cow on day two after surgery and remained in place for four days after surgery. The bandage (but not the sutures) was then removed. Follow-up examination for three months revealed that self-sucking behavior had completely resolved in all of the treated animals.
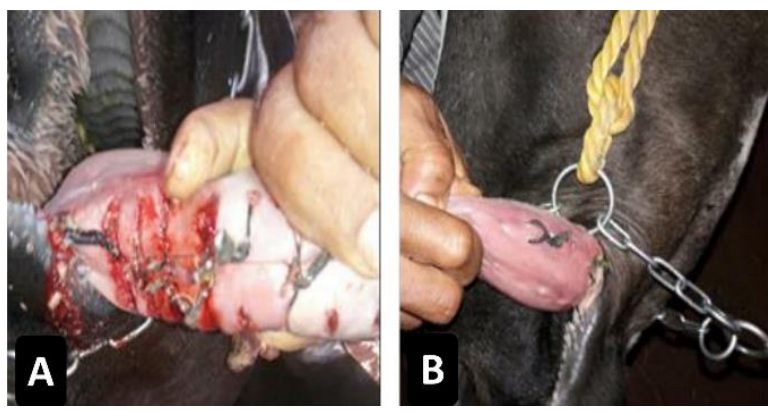

Fig. 2. The healing process after one-shot tongue reshaping. (A): Stitches are in place with superficial transverse pressure wounds at 10 days after the procedure. (B): The tongue after complete healing.

All cows are able to eat and drink effectively following the procedure. They may initially have some difficulty, but quickly adapt.

Although, stitches were designated not to be removed. Formation of pressure wounds and further primary healing of this wounds gave us an extra offer to remove these sutures after complete healing.

\section{Discussion}

Although some conservative measures may help preventing self-sucking temporarily, they remain economically in effective, as, the affected animal became market inferior so as these methods are used. Another major problem of the conservative remedies is that it may stop self-sucking but never stops development of inter-sucking problem.

Surgical intervention is the most reliable solution for self-sucking behavior in dairy cattle (Abou-El-Ella, 1999; Ducharme, 2004; Bademkiran et al., 2006). Ventral and partial glossectomy procedures both include excision and suturing of the lingual tissue. By contrast, the one-shot tongue reshaping technique does not require lingual resection. Several complications accompany the former techniques, such as bleeding and infection (Yong et al., 2008). The present technique is minimally invasive. The lingual blood vessels are not incorporated in the suture and the lingual tissue is only marginally invaded. The previously reported surgical techniques (McCormack, 1976; Dietz and Ludwig, 1979; Kersjes et al., 1985; Bademkiran et al., 2006; Yong et al., 2008) and the one-shot suturing technique provide similar results.

The dorsal surface of the tongue becomes convex, and cupping is prevented. From our point of view, the ventral glossectomy technique has a major challenge which is the determination of accurate size and thickness of the tongue mucosa to be excised. The suturing technique presented here was easy to perform and did not require mucosal resection.

Complications following a partial glossectomy are significant. As contamination is inevitable, infection rate gets high. 
Excessive swelling of the tongue and suture dehiscence are other two possible post-operative complications. The current technique involves minimal tissue invasion and as a result no concurrent complications were noted except for swelling which diminished few days after the procedure.

The current technique offers a great control to iatrogenic complications over the ventral glossectomy techniques. Stitches may be removed any time and the procedure could be performed again in right manner. In conclusion, one-shot tongue reshaping technique is reliable, easy, effective, and time saving, that elect this technique to have wider applicability especially in those areas with high incidence rate of self-sucking. Feasibility, field applicability and minimal tissue invasiveness if the current technique give the veterinary surgeons an extra safety options. Some additional research that would develop the ideas further would be recommended.

\section{Conflict of interest}

The authors declare that there is no conflict of interests.

\section{References}

Abou-El-Ella, A. 1999. Surgical Treatment of Anomalous Milk Sucking in Friesian Dairy Cattle. Assiut Vet. Med. J. 42, 260-270.

Allmacher, G. 1998. Mouth-nose ring to prevent cattle from sucking. Dtsch Tierarztl Wochenschr. 105(3), 104-105.

Bademkiran, S., Celik, R., Yesilmen, S., Kanay, B. and Kilinc, M. 2007. The effects of self-sucking on daily milk product, udder health and the form of the teats of dairy cows. J. Anim. Vet. Adv. 6(11), 1250-
1254.

Bademkiran, S., Üstün, B. and Ersöz Kanay, B. 2006. Application of Some Methods to Prevent of SelfSucking in Dairy Cattle in Diyarbakır Province. YYU Vet. Fak. Derg. 17(1-2), 59-64.

Boe, K. 1990. Suction problem in loose farm cattle. Currently from the state's press service for agriculture, Information Meeting Technique. 7, 348-353.

Debreceni, O. and Juhas, P. 1999. Milk-sucking in dairy cattle in loose housing in Slovakia. Livestock Prod. Sci. 61, 1-6.

Dietz, O. and Ludwig, P. 1979. Surgical treatment of milk suckers (self-sucking cows). Monatshefte für Veterinärmedizin, 34(11), 417-420.

Ducharme, N. 2004. Surgery of the bovine digestive system. Farm animal surgery, Fubini \& Duchrane, Elsevier, (USA).

Kersjes, A., Nemeth, F. and Rutgers, L. 1985. Lingual mucosa resection in cattle. Atlas of large animal surgery, Williams \& Wilkins, USA.

Lyle, C. 1971. Tongue surgery prevents sucking habit in mature cow. Hoard's Dairyman, 116, 1054.

McCormack, J. 1976. Surgical procedure for prevention of self-sucking in cattle (a photographic essay). Vet. Med. Small Anim. Clin. 71(5), 681683.

Pamer, F. 1981. Prevention of Sucking in Cows by a Lingual Operation. Magyar Allatorvosok Lapja. 36, 348-350.

Yong, H., Cho, J. and Kim, S. 2008. Tongue surgery of a cow showing a constant inter sucking behavior. J. Vet. Clin. 25(2), 136-138. 